\title{
Cinética de secagem e difusividade efetiva em folhas de jenipapo (Genipa americana L.)
}

SILVA, L.A. ${ }^{* *}$; RESENDE, O.1; VIRGOLINO, Z.Z.'; BESSA, J.F.V.'; MORAIS, W.A.'; VIDAL, V.M. ${ }^{1}$ Instituto Federal de Educação, Ciência e Tecnologia Goiano, Câmpus Rio Verde - Rod. Sul Goiana Km 01, Zona Rural, CEP 75.901-970, Caixa Postal 66, Rio Verde, GO - Brasil. *Autor para correspondência: lilianabadia5@ gmail.com

RESUMO: O jenipapo (Genipa americana L.) é uma espécie nativa com importância medicinal, sendo amplamente utilizada no Brasil. Em função da necessidade de conhecimento à cerca do pré-processamento desta espécie, este trabalho foi desenvolvido com o objetivo de avaliar a cinética de secagem de folhas de jenipapo (G. americana L.), bem como determinar a difusividade efetiva da água durante o processo. As folhas foram coletadas com teor de água inicial de 2,30 $\pm 0,05$ (decimal b.s.), e submetidas à secagem em três condições de temperatura do $\operatorname{ar}\left(35,3 ; 46,0\right.$ e $\left.65,0^{\circ} \mathrm{C}\right)$ até atingirem o teor de água de equilíbrio. Aos dados experimentais, ajustaram-se doze modelos matemáticos, recomendados para representar 0 processo de secagem de produtos agrícolas. As magnitudes do coeficiente de determinação $\left(R^{2}\right)$, do erro médio relativo $(P)$, do erro médio estimado $(S E)$ e do teste do qui-quadrado $\left(X^{2}\right)$, foram utilizadas para verificar o grau de ajuste dos modelos. Os modelos de Henderson e Pabis modificado e Midilli apresentaram ajustes adequados aos dados experimentais, sendo o modelo de Midilli, em função de sua simplicidade, escolhido para representar a cinética de secagem das folhas de jenipapo. Aumentando a temperatura do ar de secagem de 35,3 para 46,0 e $65,0^{\circ} \mathrm{C}$ houve redução no tempo de secagem das folhas de jenipapo de 91,1 para 62,5 e 24,2 horas, respectivamente. O coeficiente de difusão efetivo aumenta com a elevação da temperatura, e esta relação é descrita pela equação de Arrhenius, que apresenta energia de ativação para a difusão líquida de 33,9 kJ mol-1.

Palavras-chave: Modelo de Midilli, desidratação, plantas medicinais, energia de ativação

\begin{abstract}
Drying kinetics and effective diffusivity in jenipapo sheets (Genipa americana L.). The jenipapo (Genipa americana L.) is a native species with medicinal importance and is widely used in Brazil. Due to the need for knowledge about the preprocessing of this species, this work was developed to evaluate the drying kinetics of the jenipapo leaves ( $G$. americana L.), and also to determine the effective diffusivity of water during the process. The leaves were collected with an initial moisture content of $2.30 \pm 0.05$ (decimal db), and dried in three conditions of air temperature $\left(35.3,46.0\right.$ and $\left.65.0^{\circ} \mathrm{C}\right)$ until they reach the equilibrium moisture content. The experimental data set were twelve mathematical models, recommended to represent the drying process of agricultural products. The magnitudes of the coefficient of determination $\left(R^{2}\right)$, the mean relative error $(P)$, the average estimated error $(S E)$ and the chi-square $\left(X^{2}\right)$, were used in order to verify the adequacy level of the models. The Henderson, modified Pabis and Midilli models presented appropriate adjustments to the experimental data, with the model Midilli, due to its simplicity, chosen to represent the drying kinetics of the jenipapo leaves. By increasing the temperature of the drying air from 35.3 to 46.0 and $65.0^{\circ} \mathrm{C}$, there was a reduction in the drying time of the jenipapo leaves, from 91.1 to 62.5 and to 24.2 hours, respectively. The effective diffusion coefficient increases with the temperature's raise, and this relationship is described by the Arrhenius equation, which shows activation energy for liquid diffusion of $33.9 \mathrm{~kJ} \mathrm{~mol}^{-1}$.
\end{abstract}

Keywords: Model Midilli, dehydration, medicinal plants, activation, energy. 


\section{INTRODUÇÃO}

O Jenipapo (Genipa americana L.) é uma espécie pertencente à família Rubiaceae, de ocorrência em todos os estados do Brasil, em várias formações florestais situadas em várzeas úmidas ou encharcadas (Lorenzi, 2008).

Além da importância ecológica e econômica desta espécie, destaca-se o uso medicinal, com diversas partes da planta sendo utilizadas na medicina caseira em muitas regiões do país. Os frutos, as folhas e as cascas são indicadas em casos de tosse, anemia, contusões, luxações, bem como atividade purgativa, diurética e depurativa. Nas folhas e nos frutos são encontradas quantidades satisfatórias de iridóides, taninos, ácido geniposídico e genipacetol, com ação farmacológica antiulcerogênica, anticarcinogênica, antidiarreica, antigonorréica, antiasmático, antianêmico, antiinflamatória e antioxidante (Lorenzi \& Matos, 2008; Souza et al., 2013). A infusão das folhas é utilizada contra doenças do fígado, antidiarreico e, quando macerada, como febrífugo (Erbano \& Duarte, 2010).

A parte aérea das plantas medicinais, principal fonte de produtos fitoterápicos, é colhida geralmente com alto teor de água, o que ocasiona o aumento de atividades metabólicas, resultando em alterações químicas e físicas no produto quando armazenado. Neste sentido, a secagem de plantas medicinais e aromáticas, é fundamental para o controle e a manutenção da qualidade do produto final, uma vez que as indústrias farmacêuticas de fitoterápicos possuem baixa capacidade de processamento de produtos úmidos, e por isso precisam armazenar este material (Goneli et al., 2014; Lorenzi \& Matos, 2008). O processo de secagem, em condições constantes de temperatura, umidade relativa e velocidade do ar é dividido em período de velocidade constante, em que a temperatura do produto se mantém em equilíbrio com a temperatura do ar de secagem saturado, compensando as transferências de calor e massa, e o período de velocidade decrescente, em que a taxa de transporte interno de água é menor do que a taxa de evaporação (Brooker et al., 1992).

Os métodos e as condições de secagem devem ser adequados as características de cada tipo de produto. Neste sentido, torna-se necessária a obtenção de informações teóricas sobre o comportamento de cada produto durante a secagem. Tais informações podem ser obtidas por meio da simulação da secagem utilizando modelos matemáticos para descrever o processo de perda de água (Afonso Júnior \& Corrêa, 1999; Martinazzo et al., 2010; Resende et al., 2008). Neste sentido, os modelos matemáticos são fundamentais para o desenvolvimento e aperfeiçoamento de equipamentos utilizados para a secagem, bem como para estimar o tempo necessário para a redução do teor de água do produto (Andrade et al., 2003; Berbert et al., 1995).

São propostos três tipos de modelos para predizer o comportamento da secagem: os modelos teóricos, os modelos semiteóricos e os empíricos. Destes, os semiteóricos e empíricos, que consideram somente a resistência externa à temperatura e umidade relativa do ar de secagem, são considerados mais adequados para predizer 0 processo de redução de água (Brooker et al., 1992). O modelo da difusão líquida é o modelo teórico mais intensamente investigado e se baseia na segunda Lei de Fick, que descreve que o fluxo de massa por unidade de área é proporcional ao gradiente de concentração de água (Park et al., 2002).

Estudos sobre a cinética de secagem em camada delgada, são realizados com diversos produtos agrícolas, como sementes, grãos, frutos, espécies de plantas medicinais, aromáticas e condimentares, com ajuste de diferentes modelos matemáticos, conforme o produto e as condições do processo de secagem (Martinazzo et al., 2007). $\mathrm{O}$ ajuste de modelos matemáticos aos dados experimentais de secagem e as características de cada espécie, são de grande importância na tomada de decisão e contribuem na melhoria da eficiência do processo de secagem (Radünz et al., 2011).

Recentemente diversos trabalhos têm sido desenvolvidos para investigar o comportamento, durante a secagem de diferentes espécies de plantas medicinais e aromáticas, por meio do ajuste de modelos matemáticos (Akpinar, 2006; Doymaz, 2006; Martinazzo et al., 2007; Martinazzo et al., 2010; Dias et al., 2011; Doymaz, 2011; Radünz et al., 2011; Prates et al., 2012; Reis et al., 2012; Oliveira et al., 2013; Doymaz, 2014; Rodríguez et al., 2014)and air velocity of $1 \mathrm{~m} / \mathrm{s}$. A theoretical diffusion model and eight different empirical models were fitted to the experimental data. From the theoretical model application, the effective diffusivity per unit area of the thyme was estimated (between 3.68 I u00d7 10(-5. No entanto, não foram encontrados na literatura trabalhos que retratem o comportamento das folhas de jenipapo (Genipa americana L.) quando submetidas a diferentes temperaturas do ar de secagem.

Assim, objetivou-se com este trabalho analisar a cinética de secagem de folhas de jenipapo (Genipa americana L.) para diferentes temperaturas do ar de secagem, bem como ajustar modelos matemáticos, determinar o coeficiente de difusão efetivo e a energia de ativação durante o processo. 


\section{MATERIAL E MÉTODOS}

O experimento foi realizado no Laboratório de Pós-colheita de Produtos Vegetais do Instituto Federal de Educação, Ciência e Tecnologia Goiano - Câmpus Rio Verde, Goiás, Brasil (IF GoianoCâmpus Rio Verde).

As folhas de jenipapo (Genipa americana L.) foram coletadas de uma árvore matriz situada no bosque do IF Goiano Câmpus Rio Verde $\left(17^{\circ}\right.$ $\left.48^{\prime} 945^{\prime \prime} \mathrm{S}-50^{\circ} 54^{\prime} 2219^{\prime \prime} \mathrm{W}\right)$. Após a coleta, as folhas foram acondicionadas em sacos plásticos de polietileno e encaminhadas ao laboratório.

A secagem foi realizada em um secador de leito fixo, construído com chapa número 16 , com câmara de secagem de 0,60 × 0,60 × 0,60 $\mathrm{m}$ de volume total de $0,216 \mathrm{~m}^{3}$ e uma placa com $25 \%$ de perfuração localizada a uma altura de $0,33 \mathrm{~m}$. O ventilador acoplado é do tipo centrífugo e impulsionado por um motor trifásico com uma potência de $1,5 \mathrm{cv}$ a $1.720 \mathrm{rpm}$, consistindo de um rotor, lâminas, uma voluta e um suporte, estando ligado à câmara de secagem por um elemento de expansão que converte a seção de $0,20 \times 0,20 \mathrm{~m}$ na saída do ventilador para $0,57 \times 0,03 \mathrm{~m}$ na entrada do câmara de secagem ao longo de um comprimento de 0,64 m (Figura 1)

Cada câmara de secagem é composta por 6 sensores de temperatura pendulares e quatro resistências elétricas de 1.500 watts, perfazendo um total de 6.000 watts. Os sensores foram posicionados antes e após o sistema de aquecimento e no interior de cada bandeja. Na câmara de secagem foram colocadas quatro bandejas removíveis com fundo perfurado, com dimensões de 0,28 x 0,28 x 0,15 m, para permitir a passagem do ar através da camada de produto, perfazendo, para cada temperatura de secagem utilizada, quatro repetições.

As temperaturas do ar ambiente e da sala de secagem foram monitoradas com termopares instalados interna e externamente ao ambiente de secagem. A umidade relativa dentro do secador foi obtida utilizando-se os princípios básicos de psicrometria com auxílio do software GRAPSI.

A temperatura média do ar ambiente foi $26,3 \pm 1,1^{\circ} \mathrm{C}$ e a umidade relativa de $72,3 \pm 2,3 \%$ durante todo o período de secagem. A velocidade do ar utilizada para a secagem foi de $1,0 \mathrm{~m} . \mathrm{s}^{-1}$ regulada com auxílio de um anemômetro de pás rotativas. $O$ sistema foi regulado para aquecer a 35,3; 46,0 e $65,0^{\circ} \mathrm{C}$, resultando nas umidades relativas do ar de secagem de 42,0; 24,4 e 9,9\%, respectivamente.

Para a secagem, foram envolvidos $250 \mathrm{~g}$ de folhas de jenipapo por tecido do tipo voile e espalhados sobre as bandejas do secador formando uma camada de aproximadamente 3,0 $\mathrm{cm}$, sendo quatro repetições por temperatura. $\mathrm{O}$ teor de água inicial das folhas de jenipapo foi de $2,30 \pm 0,05$ (decimal b.s.), sendo que a secagem prosseguiu até que as folhas alcançassem o teor de água de equilíbrio com o ar, em cada temperatura de secagem. Os teores de água, antes e após a secagem, foram determinados pelo método gravimétrico recomendado pela Asae (2000), para forragens e folhas, em estufa com circulação forçada de ar a $103 \pm 1^{\circ} \mathrm{C}$, durante 24 horas. A redução do teor de água ao longo da secagem foi acompanhada pelo método gravimétrico (perda de massa), por meio de pesagens das folhas durante o processo de secagem, conhecendo-se o teor de água inicial até atingir o teor de água de equilíbrio, utilizandose uma balança digital com $0,01 \mathrm{~g}$ de resolução. O tempo entre as leituras foi controlado por meio da diferença de massa, de modo a não permitir diferenças acentuadas de teor de água durante as leituras.

Para a determinar a razão de teor de água

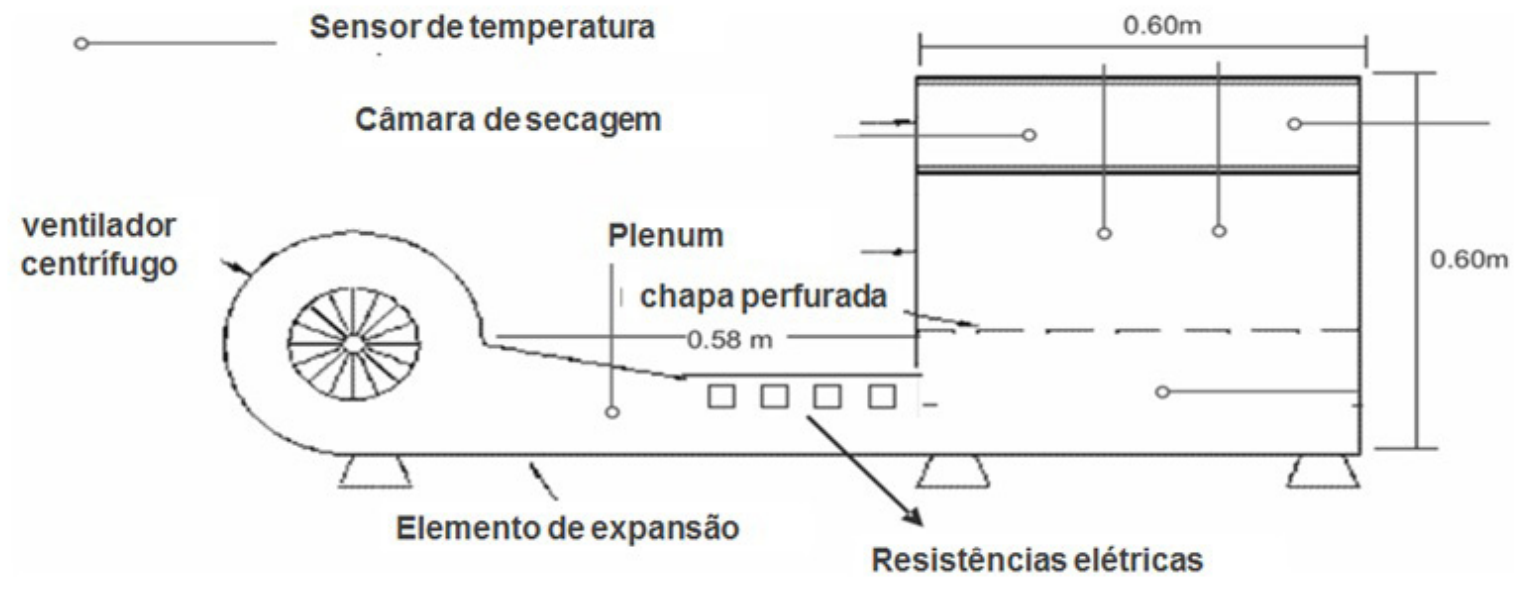

FIGURA 1. Vista lateral da câmara de secagem experimental. (Laboratório de Pós-Colheita de Produtos Naturais, Instituto Federal Goiano, Câmpus Rio Verde, Rio Verde - GO).

Rev. Bras. PI. Med., Campinas, v.17, n.4, supl. II, p.953-963, 2015. 
das folhas de jenipapo durante o processo de secagem, utilizou-se a seguinte expressão:

$$
R X=\frac{X^{*}-X_{e}^{*}}{X^{*}{ }_{i}-X_{e}^{*}}
$$

em que:

$\mathrm{RX}$ : razão de teor de água do produto (adimensional); $\mathrm{X}^{*}$ : teor de água do produto (decimal b.s.);

$\mathrm{X}^{*}$ : teor de água inicial do produto (decimal b.s.);

$\mathrm{X}_{\mathrm{e}}^{*}$ : teor de água de equilíbrio do produto (decimal b.s.)
Aos dados experimentais de razão de teor de água, durante a secagem das folhas de jenipapo, foram ajustados doze modelos matemáticos frequentemente utilizados para representação do fenômeno de secagem de produtos agrícolas, conforme Tabela 1.

Os modelos matemáticos foram ajustados por meio de análise de regressão não-linear pelo método Gauss Newton. Os modelos foram selecionados considerando a magnitude do desvio padrão da estimativa (SE), coeficiente de determinação $\left(R^{2}\right)$, do erro médio relativo $(P)$ e do

TABELA 1. Modelos matemáticos utilizados para predizer o fenômeno de secagem de produtos agrícolas.

\begin{tabular}{|c|c|c|}
\hline Designação do modelo & Modelo & \\
\hline Wang e Singh (Wang \& Sing 1978) & $R X=1+a t=b t^{2}$ & $(2)$ \\
\hline Verma (Verma et al. 1985) & $R X=a \cdot \exp (-k \cdot t)+(1-a) \exp \left(-k_{1} \cdot t\right)$ & (3) \\
\hline Thompson (Thompson et al. 1968) & $R X=\exp \left(\left(-a-\left(a^{2}+4 \cdot b \cdot t\right)^{0,5}\right) / 2 \cdot b\right)$ & $(4)$ \\
\hline Page (Page 1949) & $R X=\exp \left(-k \cdot t^{n}\right)$ & (5) \\
\hline Newton (Lewis 1921) & $R X=\exp (-k \cdot t)$ & $(6)$ \\
\hline Midilli (Midilli et al. 2002) & $\left.R X=a \cdot \exp \left(-k \cdot t^{n}\right)+b \cdot t\right)$ & $(7)$ \\
\hline Logarítmico (Yagcioglu et al. 1999) & $R X=a \cdot \exp (-k \cdot t)+c$ & $(8)$ \\
\hline Henderson e Pabis (Henderson \& Pabis 1961) & $R X=a \cdot \exp (-k \cdot t)$ & $(9)$ \\
\hline Henderson e Pabis Modificado (Karathanos 1999) & $R X=a \cdot \exp (-k \cdot t)+b \cdot \exp \left(-k_{o} \cdot t\right)+c \cdot \exp \left(-k_{1} \cdot t\right)$ & $(10)$ \\
\hline Exponencial de Dois Termos (Sharaf-Eldeen et al. 1980) & $R X=a \cdot \exp (-k \cdot t)+(1-a) \exp (-k \cdot a \cdot t)$ & $(11)$ \\
\hline Dois Termos (Henderson 1974) & $R X=a \cdot \exp \left(-k_{o} \cdot t\right)+b \cdot \exp \left(-k_{1} \cdot t\right)$ & $(12)$ \\
\hline Aproximação da Difusão & $R X=a \cdot \exp (-k \cdot t)+(1-a) \cdot \exp (-k \cdot b \cdot t)$ & $(13)$ \\
\hline
\end{tabular}

t: tempo de secagem (horas); $k, k_{0}, k_{1}$ : constantes de secagem ( $\left.h-1\right) ; a, b, c, n$ : coeficientes dos modelos.

teste de qui-quadrado $\left(\mathrm{X}^{2}\right)$. Considerou-se o valor do erro médio relativo inferior a $10 \%$ como um dos critérios para seleção dos modelos, de acordo com Mohapatra \& Rao (2005). Os erros médios relativo e estimado e o desvio padrão, para cada um dos modelos, foram calculados conforme as seguintes expressões:

$$
\begin{aligned}
& \mathrm{P}=\frac{100}{\mathrm{~N}} \sum \frac{|\mathrm{Y}-\hat{\mathrm{Y}}|}{\mathrm{Y}} \\
& \mathrm{SE}=\sqrt{\frac{\sum(\mathrm{Y}-\hat{\mathrm{Y}})^{2}}{\mathrm{GLR}}} \\
& \chi^{2}=\frac{\sum_{i=1}^{\mathrm{n}}(\mathrm{Y}-\hat{\mathrm{Y}})^{2}}{\mathrm{GLR}}
\end{aligned}
$$

em que:

Y: valor observado experimentalmente; $\hat{Y}$ : valor estimado pelo modelo;
$\mathrm{N}$ : número de observações experimentais;

GLR: graus de liberdade do modelo (número de observações experimentais menos o número de coeficientes do modelo).

O coeficiente de difusão efetivo (D) para a secagem das folhas de jenipapo, para as diferentes condições de secagem, foi calculado por meio da Equação 17, com base no modelo matemático da difusão líquida com a solução analítica para a placa plana infinita, com aproximação de oito termos:

$$
R X=\frac{X^{*}-X_{e}^{*}}{X_{i}^{*}-X_{e}^{*}}=\frac{8}{\pi^{2}} \sum_{n=0}^{\infty} \frac{1}{(2 n+1)^{2}} \exp \left[-\frac{(2 n+1)^{2} \cdot \pi^{2} \cdot D \cdot t}{4} \cdot\left(\frac{S}{V}\right)^{2}\right]
$$

em que:

RX: razão de teor de água do produto (adimensional);

$\mathrm{n}$ : número de termos;

S: área da superfície da folha $\left(\mathrm{m}^{2}\right)$;

$\mathrm{V}$ : volume da folha, $\left(\mathrm{m}^{3}\right)$.

O volume das folhas de jenipapo foi obtido por meio da relação entre as medidas dos três eixos ortogonais $\left(a_{i}\right.$ : comprimento; $b_{i}$ largura e $c_{i}$

Rev. Bras. PI. Med., Campinas, v.17, n.4, supl. II, p.953-963, 2015. 
espessura). Para determinação da largura e da espessura das folhas de jenipapo, obteve-se a média dos valores de 15 medições ao longo do limbo foliar de 15 folhas totalmente expandidas, utilizando-se paquímetro digital com resolução de $0,01 \mathrm{~mm}$. A partir destes valores, o volume das folhas de jenipapo foi calculado utilizando-se a seguinte expressão (Mohsenin, 1986):

$$
V f=\frac{\pi \cdot\left(a_{i} \cdot b_{i} \cdot c_{i}\right)}{6}
$$

em que,

$\mathrm{V}_{\mathrm{f}}$ : volume da folha, $\mathrm{m}^{3}$;

$a_{1}$ : dimensão do maior eixo da folha $(m)$;

$b_{i}$ : dimensão do eixo médio da folha $(m)$;

$c_{i}$ : dimensão do menor eixo da folha $(m)$.

A área superficial das folhas de jenipapo foi calculada por meio das seguintes expressões:

$$
\begin{gathered}
\mathrm{S}=\pi \cdot \mathrm{D}_{\mathrm{g}} \\
D_{g}=\left(a_{i} \cdot b_{i} \cdot c_{i}\right)^{1 / 3}
\end{gathered}
$$

em que:

$\mathrm{S}$ : área superficial $\left(\mathrm{m}^{2}\right)$;

$\mathrm{D}_{\mathrm{g}}$ : diâmetro geométrico médio $(\mathrm{m})$

Para avaliar a influência da temperatura no coeficiente de difusão efetivo, foi utilizada a equação de Arrhenius, descrita da seguinte forma:

$$
\mathrm{D}=\mathrm{D}_{\mathrm{o}} \cdot \exp \left(\frac{\mathrm{E}_{\mathrm{a}}}{\mathrm{R} \cdot \mathrm{T}_{\mathrm{a}}}\right)
$$

em que:

$\mathrm{D}$ : coeficiente de difusão líquida efetiva, $\mathrm{m}^{2} \mathrm{~s}^{-1}$;

$D_{0}$ : fator pré-exponencial;

$\mathrm{E}_{\mathrm{a}}$ : energia de ativação $\left(\mathrm{kJ} \mathrm{mol}^{-1}\right)$;

$\mathrm{R}$ : constante universal dos gases $\left(8,314 \mathrm{~kJ} \mathrm{kmol}^{-1} \mathrm{~K}^{-1}\right)$;

$\mathrm{T}_{\mathrm{a}}$ : temperatura absoluta $(\mathrm{K})$.

Os coeficientes da expressão de Arrhenius foram obtidos linearizando-se a Equação $21 \mathrm{com}$ a aplicação do logaritmo neperiano, da seguinte forma:

$$
\mathrm{LnD}=\mathrm{LnD}_{\mathrm{o}}-\frac{E_{a}}{R} \cdot \frac{1}{\mathrm{~T}_{\mathrm{a}}}
$$

\section{RESULTADOS E DISCUSSÃO}

Nas Tabelas 2 e 3 são apresentados os parâmetros estatísticos usados para comparar os 12 modelos testados para descrever a cinética de secagem de folhas de jenipapo. Para as temperaturas de 35,3 e $65,0^{\circ} \mathrm{C}$, os modelos matemáticos ajustados aos dados experimentais apresentaram coeficiente de determinação $\left(R^{2}\right)$ superior a $92,68 \%$, fato não observado para a temperatura de $46,0^{\circ} \mathrm{C}$, em que alguns modelos tiveram valores inferiores.

A maioria dos modelos testados, para as três temperaturas, apresentaram valores de erro médio relativo superiores a $10 \%$, demonstrando que tais modelos não devem ser utilizados para explicar o fenômeno de secagem das folhas de jenipapo. Aliado a este parâmetro, optou-se por adotar um modelo que apresente bom ajuste para todas as temperaturas testadas.

A partir da análise das Tabelas 2 e 3 observase que os modelos de Verma, Logaritmo, Henderson e Pabis, Exponencial de dois termos e Aproximação da Difusão se ajustaram satisfatoriamente aos dados de secagem nas temperaturas de 35,3 e $65,0^{\circ} \mathrm{C}$.

O modelo de Midilli foi o único que apresentou baixo valor de erro médio estimado (SE), com coeficiente de determinação acima de $99 \%$, qui-quadrado significativo e valor de erro médio relativo inferior a $10 \%$ para as três temperaturas testadas, mostrando ajuste adequado aos dados experimentais, e podendo representar o fenômeno de secagem das folhas de jenipapo nas diferentes condições testadas. Assim, o modelo de Midilli foi selecionado para representar a cinética de secagem das folhas de jenipapo, conforme ilustrado na Figura 2.

De um modo geral, o modelo de Midilli apresenta um bom ajuste os dados de secagem de folhas de plantas medicinais, aromáticas ou condimentares, visto que, este também foi o modelo de melhor ajuste aos dados experimentais de secagem de folhas de aroeira (Goneli et al., 2014), louro (Doymaz, 2014), fruta-de-lobo (Prates et al., 2012), manjericão (Reis et al., 2012), carqueja (Radünz et al., 2011), alecrim (Arslan \& Musa Özcan, 2008) e capim-limão (Martinazzo et al., 2007). Segundo Goneli et al. (2014), nos estádios iniciais do processo de secagem de plantas medicinais ocorre rápida perda de água, caracterizando uma curva mais acintosa, o que justifica o ajuste do modelo de Midilli aos dados experimentais. No entanto, segundo Radünz et al. (2011) o ajuste do modelo matemático depende das características de cada espécie vegetal, sendo necessária a realização de estudos individuais para as diversas espécies.

$\mathrm{Na}$ Tabela 4 são apresentados os coeficientes do modelo de Midilli ajustados aos dados

Rev. Bras. PI. Med., Campinas, v.17, n.4, supl. II, p.953-963, 2015. 
TABELA 2. Erro médio estimado (SE decimal), erro médio relativo $(P \%)$ e coeficientes de determinação $\left(\mathrm{R}^{2} \%\right)$ para os doze modelos analisados, durante a secagem de folhas de jenipapo em três condições de temperatura $\left({ }^{\circ} \mathrm{C}\right)$.

\begin{tabular}{llllllllll}
\hline \multirow{2}{*}{ Modelos } & \multicolumn{2}{l}{$35,3^{\circ} \mathrm{C}$} & \multicolumn{3}{c}{$46,0^{\circ} \mathrm{C}$} & \multicolumn{5}{c}{$65,0^{\circ} \mathrm{C}$} \\
\cline { 2 - 11 } & $\mathrm{SE}$ & $\mathrm{P}$ & $\mathrm{R}^{2}$ & $\mathrm{SE}$ & $\mathrm{P}$ & $\mathrm{R}^{2}$ & $\mathrm{SE}$ & $\mathrm{P}$ & $\mathrm{R}^{2}$ \\
\hline Wang e Singh & 0,081 & 65,125 & 94,77 & 0,192 & 263,362 & 68,21 & 0,092 & 79,603 & 92,68 \\
Verma & 0,009 & 6,044 & 99,93 & 0,010 & 18,535 & 89,51 & 0,007 & 4,520 & 99,95 \\
Thompson & 0,032 & 16,212 & 99,18 & 0,015 & 12,250 & 99,79 & 0,016 & 10,594 & 99,78 \\
Page & 0,024 & 12,831 & 99,55 & 0,008 & 14,335 & 99,94 & 0,010 & 5,797 & 99,91 \\
Newton & 0,036 & 11,982 & 98,94 & 0,042 & 36,713 & 95,00 & 0,024 & 11,848 & 99,46 \\
Midilli & 0,016 & 8,729 & 99,79 & 0,007 & 8,431 & 99,95 & 0,010 & 7,697 & 99,92 \\
Logarítmico & 0,017 & 5,326 & 99,79 & 0,023 & 16,918 & 99,56 & 0,016 & 6,565 & 99,77 \\
Henderson e Pabis & 0,017 & 5,344 & 99,77 & 0,027 & 28,380 & 99,34 & 0,017 & 7,489 & 99,74 \\
Henderson e Pabis Modificado & - & - & - & 0,000 & 2,902 & 99,97 & 0,020 & 1,783 & 99,97 \\
Exponencial de Dois Termos & 0,019 & 5,608 & 99,7 & 0,018 & 24,114 & 99,71 & 0,007 & 4,577 & 99,95 \\
Dois Termos & - & - & - & 0,009 & 18,222 & 99,92 & - & - & - \\
Aproximação da Difusão & 0,009 & 5,530 & 99,93 & 0,010 & 18,535 & 99,91 & 0,007 & 4,520 & 99,95 \\
\hline
\end{tabular}

TABELA 3. Valores para o teste de qui-quadrado $\left(\mathrm{X}^{2}\right.$, decimal) para os doze modelos analisados durante a secagem de folhas de jenipapo em três condições de temperatura $\left({ }^{\circ} \mathrm{C}\right)$.

\begin{tabular}{llll}
\hline Modelos & $35,3^{\circ} \mathrm{C}$ & $46,0^{\circ} \mathrm{C}$ & $65,0^{\circ} \mathrm{C}$ \\
Wang e Singh & 0,0066 & 0,036687 & 0,0085 \\
Verma & 0,0001 & 0,000097 & 0,0000 \\
Thompson & 0,0010 & 0,000235 & 0,0002 \\
Page & 0,0006 & 0,000065 & 0,0001 \\
Newton & 0,0013 & 0,001795 & 0,0006 \\
Midilli & 0,0003 & 0,000055 & 0,0001 \\
Logarítmico & 0,0003 & 0,000517 & 0,0003 \\
Henderson e Pabis & 0,0003 & 0,000754 & 0,0003 \\
Henderson e Pabis Modificado & - & 0,000000 & 0,0004 \\
Exponencial de Dois Termos & 0,0004 & 0,000328 & 0,0001 \\
Dois Termos & - & 0,000088 & - \\
Aproximação da Difusão & 0,0001 & 0,000097 & 0,0000 \\
\hline
\end{tabular}

Significativo a $5 \%$ de probabilidade

experimentais para as diferentes temperaturas do ar de secagem das folhas de jenipapo.

Nenhuma das constantes do modelo de Midilli, ajustado aos dados experimentais, apresentaram tendência definida em resposta a elevação da temperatura do ar de secagem. Por este motivo, a cinética de secagem das folhas de jenipapo não pôde ser estimada por uma equação única, para a faixa de temperatura estudada. No entanto, a constante de secagem " $k$ ", que está relacionada com a difusividade no processo de secagem, mesmo sem apresentar uma tendência clara, apresentou um suave aumento em resposta ao incremento da temperatura do ar de secagem. O valor de "n", que varia em função da temperatura e das condições de secagem, foi menor para a temperatura de $46,0^{\circ} \mathrm{C}$. Além disso, o coeficiente "a" também aumentou com a elevação da temperatura de secagem e os valores do coeficiente "b" não foram significativos pelo teste "t" para as temperaturas de 35,3 e $65,0^{\circ} \mathrm{C}$.

Na Figura 3 é apresentada a relação entre os valores de teor de água experimentais e os 


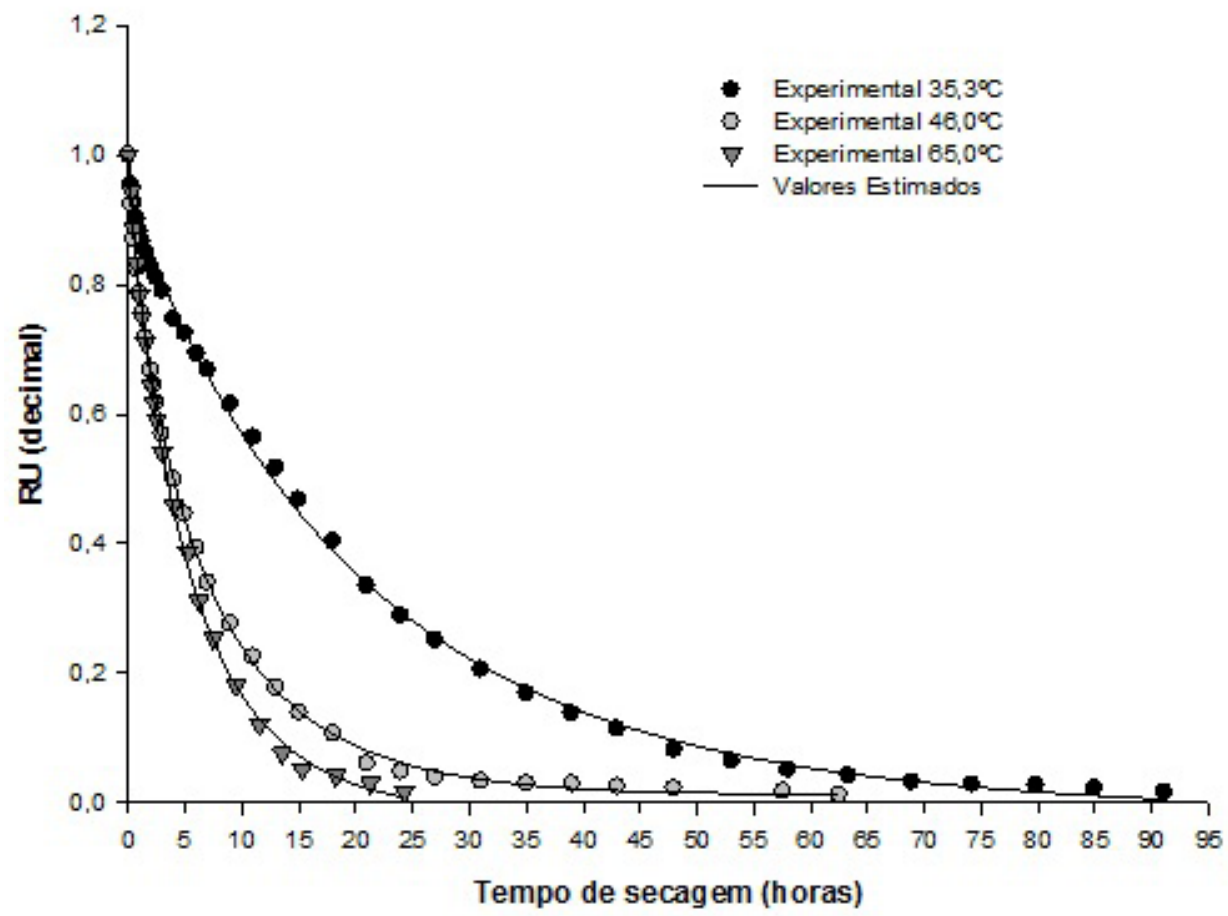

FIGURA 2. Valores da cinética de secagem das folhas de jenipapo, experimentais e estimados pelo modelo de Midilli, durante a secagem em três condições de temperatura do $\operatorname{ar}\left(35,3 ; 46,0\right.$ e $\left.65,0^{\circ} \mathrm{C}\right)$.

estimados pelo modelo de Midilli, para a secagem das folhas de jenipapo em três condições de temperatura.

O teor de água de equilíbrio, para as folhas submetidas à secagem a temperatura de $35,3^{\circ} \mathrm{C}$ foi de 0,081 (decimal b.s.), totalizando um tempo de secagem de 91,12 horas para atingir este valor. Para a temperatura de $46,0^{\circ} \mathrm{C}$ o teor de água de equilíbrio foi de 0,0486 (decimal b.s.), sendo alcançado em 62,5 horas. Já para a temperatura de $65,0^{\circ} \mathrm{C}$ foram necessárias apenas 24,2 horas até atingir o valor de teor de água de 0,0349 (decimal b.s.). Assim, aumentando a temperatura do ar de secagem de 35,3 para 46,0 ou $65,0^{\circ} \mathrm{C}$ houve redução no tempo de secagem das folhas de jenipapo em torno de 31,41 e $73,45 \%$, respectivamente.

Com o aumento da temperatura do ar de secagem houve diminuição do tempo necessário para que as folhas atingissem o teor de água de equilíbrio, evidenciando que em temperaturas mais elevadas a velocidade de retirada de água é acelerada em função da elevação da taxa de secagem. Resultados semelhantes foram obtidos em diversos trabalhos, quando se avaliou a taxa de secagem, de folhas de plantas medicinais ou aromáticas, em diferentes condições do ar de secagem, ocorrendo redução no tempo em resposta à elevação da temperatura (Goneli et al., 2014; Oliveira et al., 2013; Prates et al., 2012; Doymaz, 2014; Martinazzo et al., 2007; Doymaz, 2006).
Tabela 4. Coeficientes e constantes do modelo de Midilli ajustados ao dados das diferentes condições de temperatura do ar de secagem de folhas de jenipapo.

\begin{tabular}{|c|c|c|c|}
\hline \multirow{2}{*}{ Parâmetros } & \multicolumn{3}{|c|}{ Temperatura $\left({ }^{\circ} \mathrm{C}\right)$} \\
\hline & 35,3 & 46,0 & 65,0 \\
\hline a & $0,94926 \ldots$ & 0,99330 & 0,99848 \\
\hline $\mathrm{k}$ & 0,05820 " & $0,23150 \ldots$ & $0,23669 \cdots$ \\
\hline $\mathrm{n}$ & $0,94266^{* *}$ & 0,79014 & 0,87209** \\
\hline b & $-0,00012^{\mathrm{ns}}$ & $0,00017^{*}$ & $-0,00060^{\text {ns }}$ \\
\hline
\end{tabular}

"Significativo a $1 \%$ pelo teste t; nsNão Significativo pelo teste t

Na Figura 4 são apresentados os valores do coeficiente de difusão efetivo para as diferentes temperaturas do ar de secagem das folhas de jenipapo. Observou-se um comportamento linear crescente em que os valores do coeficiente de difusão efetivo aumentaram em resposta a elevação da temperatura do ar de secagem, variando de 1,12 $\times 10^{-12}$ a $4,02 \times 10^{-12} \mathrm{~m}^{2} \mathrm{~s}^{-1}$ para a faixa de temperatura de 35,3 a $65,0^{\circ} \mathrm{C}$.

Quando ocorre aumento na temperatura do ar de secagem, a viscosidade, que representa a resistência do fluído ao escoamento da água, diminui, o que resulta em alterações na difusão da água nos capilares das folhas, favorecendo a movimentação deste fluído no produto (Goneli et 


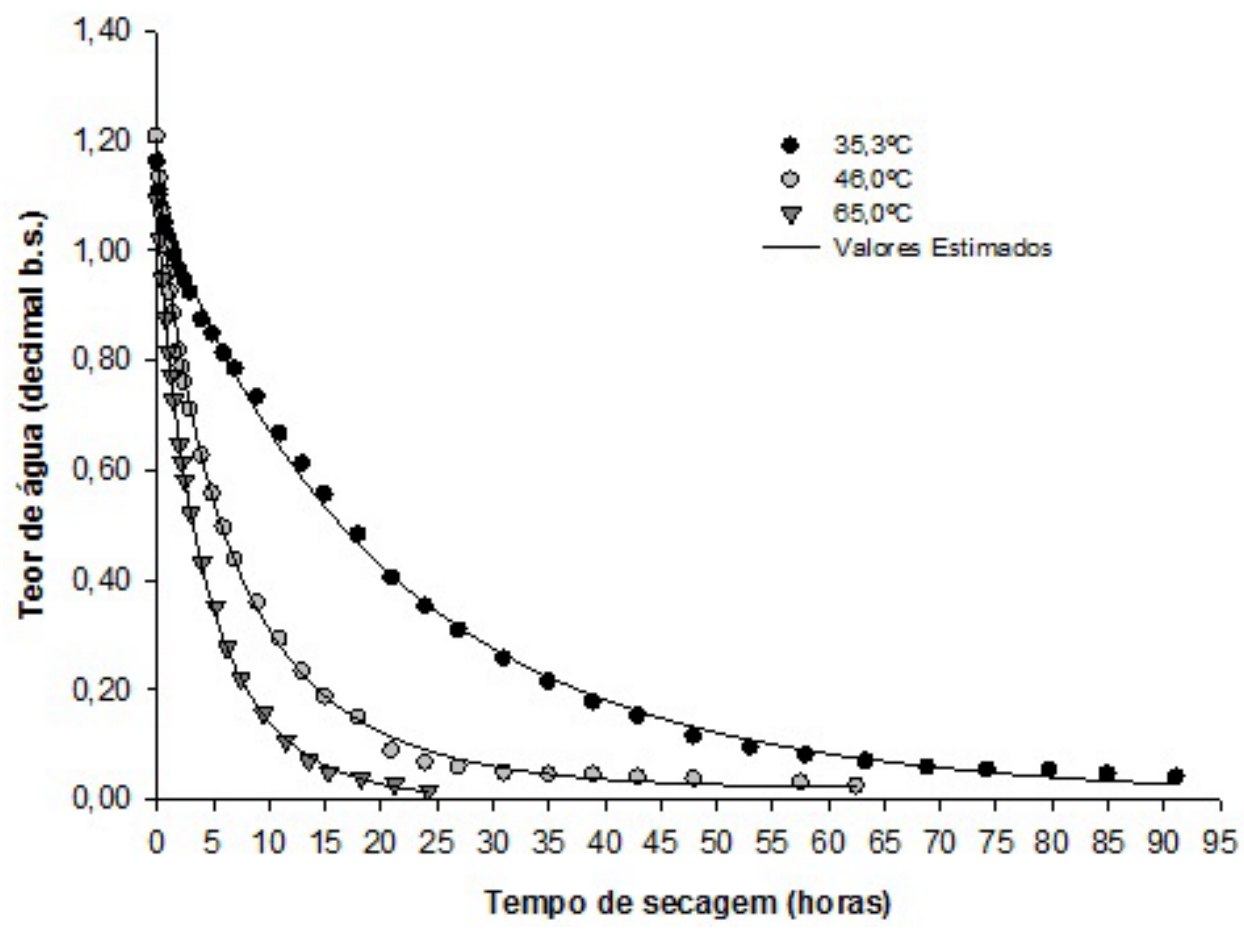

FIGURA 3. Valores de teor de água, experimentais e estimados pelo modelo de Midilli, para as folhas de jenipapo durante a secagem em três condições de temperatura do ar $\left(35,3 ; 46,0\right.$ e $\left.65,0^{\circ} \mathrm{C}\right)$.

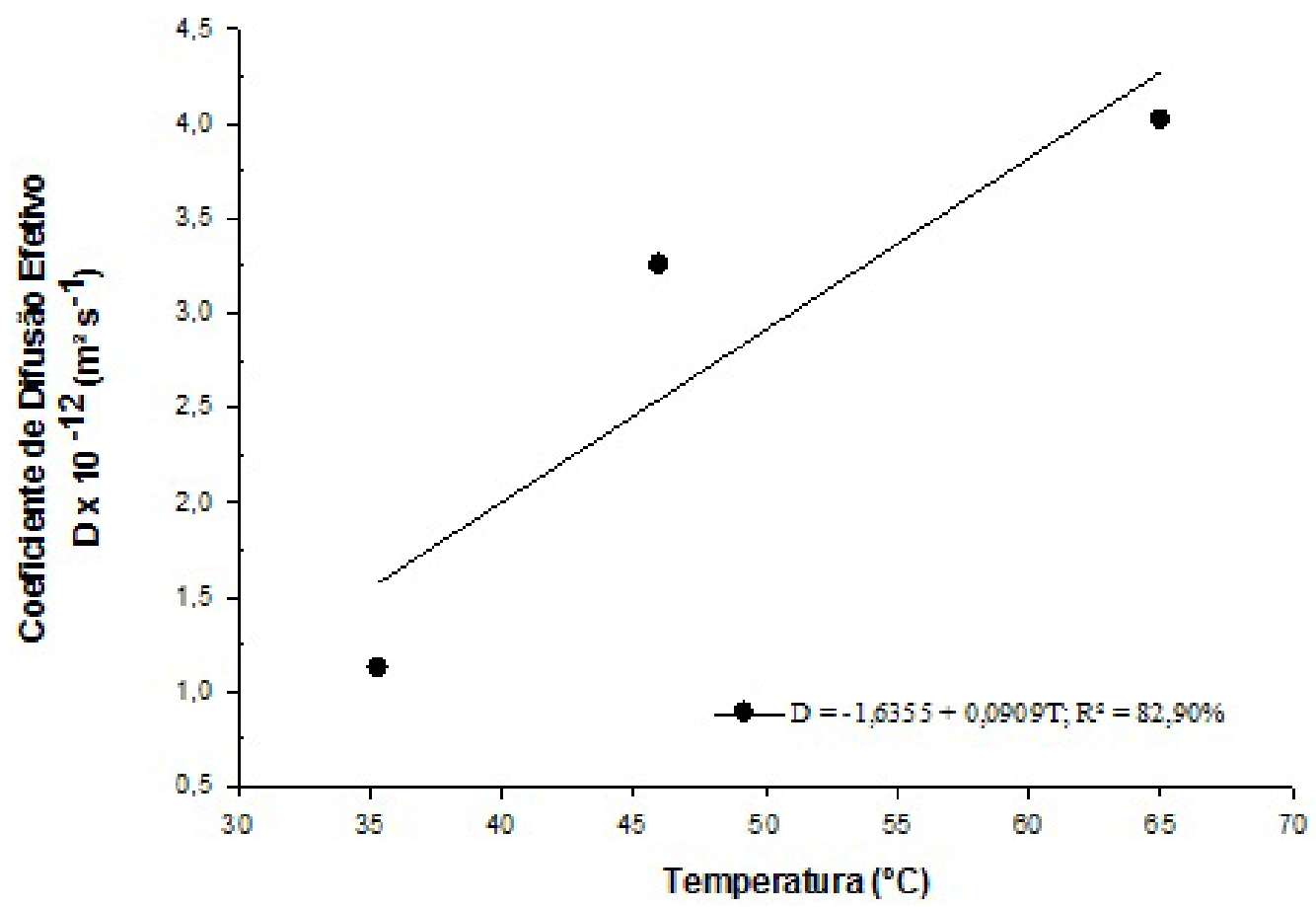

FIGURA 4. Valores médios do coeficiente de difusão efetivo $\left(\mathrm{m}^{2} \mathrm{~s}^{-1}\right)$ obtidos para a secagem de folhas de jenipapo em três condições de temperatura do $\operatorname{ar}\left(35,3 ; 46,0\right.$ e $\left.65,0^{\circ} \mathrm{C}\right)$. 


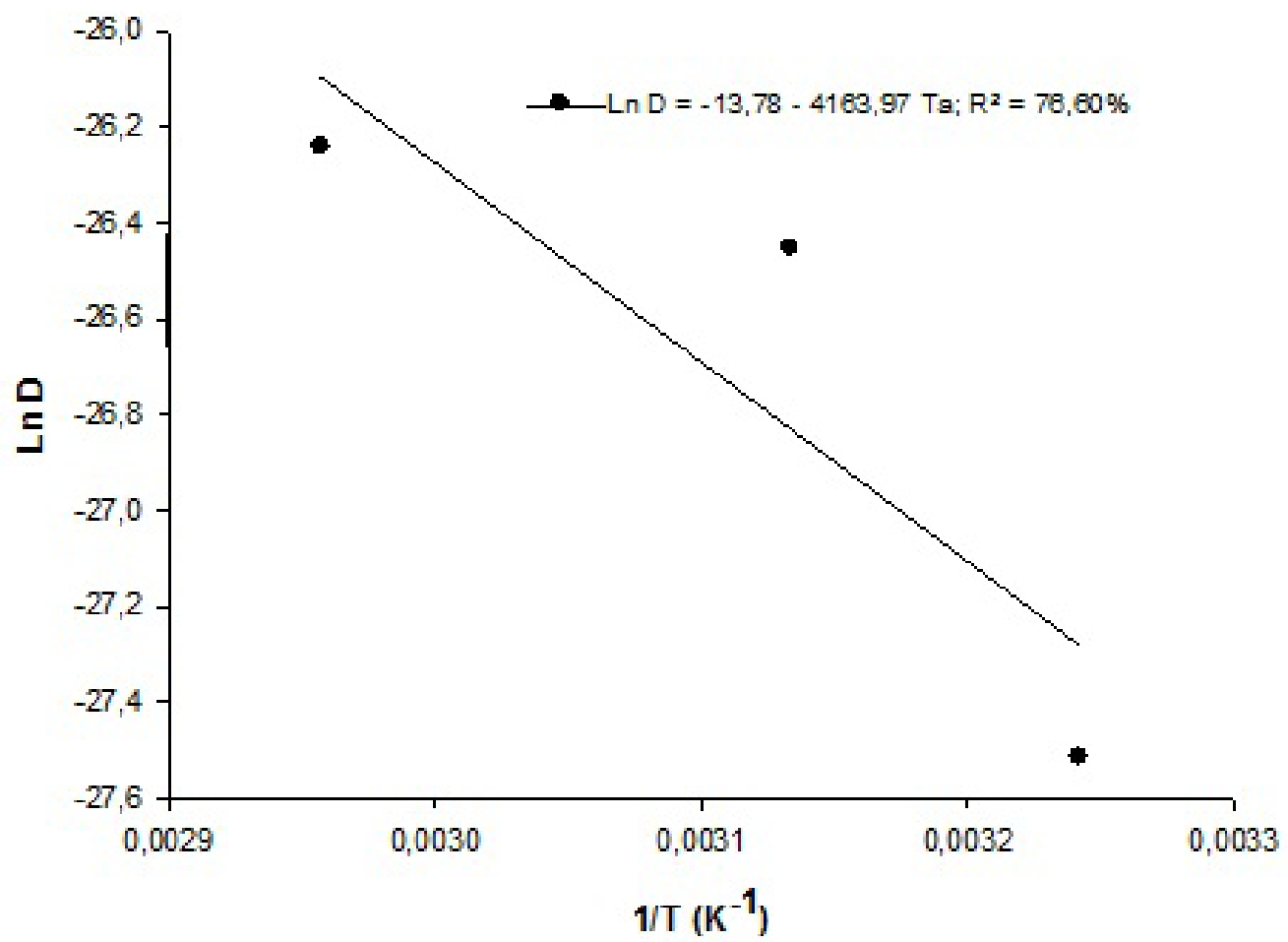

FIGURA 5. Representação de Arrhenius para o coeficiente de difusão efetivo em função da temperatura (35,3; 46,0 e $65,0^{\circ} \mathrm{C}$ ) do ar de secagem de folhas de jenipapo.

al., 2014). De acordo com Madamba et al. (1996), os valores do coeficiente de difusão efetivo para a secagem de produtos agrícolas apresentam-se na ordem de $10^{-9} \mathrm{~m}^{2} \mathrm{~s}^{-1}$ a $10^{-11} \mathrm{~m}^{2} \mathrm{~s}^{-1}$. Por outro lado, para a secagem de folhas de aroeira, Goneli et al. (2014), encontraram magnitudes de coeficientes de difusão efetivo variando entre $0,1476 \times 10^{-11} \mathrm{~m}^{2} \mathrm{~s}^{-1}$ e $1,5811 \times 10^{-11} \mathrm{~m}^{2} \mathrm{~s}^{-1}$, para a faixa de temperatura de 40 a $70^{\circ} \mathrm{C}$.

A relação entre os valores do coeficiente de difusão efetivo e a temperatura do ar de secagem é satisfatoriamente descrita pela equação de Arrhenius. A Figura 5 traz a representação de Arrhenius para o coeficiente de difusão efetivo da secagem de folhas de jenipapo, demonstrando os valores de $L n D$, em função do inverso da temperatura absoluta $\left(\mathrm{K}^{-1}\right)$.

A partir dos dados da linearização dos valores do coeficiente de difusão e da curva da representação de Arrhenius obtêm-se a relação Ea.R-1 e o valor de $D_{0}$. Desta forma, a Equação 23 apresenta a expressão de Arrhenius, ajustada para os coeficientes de difusão efetivos das folhas de jenipapo, calculada de acordo com a Equação 21:

$$
\mathrm{D}=1,04 \cdot 10^{-6} \exp \left(\frac{33,87}{\mathrm{R} \cdot \mathrm{Ta}}\right)
$$

Como observado na Equação 23, a energia de ativação para a difusão líquida no processo de secagem de folhas de jenipapo, para as três condições de temperatura estudadas (35,3; 46,0 e $65,0^{\circ} \mathrm{C}$ ) foi de $33,87 \mathrm{~kJ} \mathrm{~mol}^{-1}$. Este valor foi inferior ao encontrado para secagem de folhas de capim-limão, que apresentou energia de ativação de $63,47 \mathrm{~kJ}$ mol 1 (Martinazzo et al., 2007), folhas de fruta-de-lobo, com energia de ativação de 44, $60 \mathrm{~kJ} \mathrm{~mol}^{-1}$ (Prates et al., 2012) e para folhas de aroeira, com energia de ativação de 74,96 kJ mol-1 (Goneli et al., 2014).

Como a energia de ativação é considerada uma barreira a ser atravessada para que o processo de difusão no produto possa ocorrer (Kashaninejad et al., 2007), quanto menor a energia de ativação, maior será a difusividade de água no produto por unidade de tempo. Deste modo, o valor de energia de ativação encontrado reforça que as folhas de jenipapo apresentaram elevada difusividade durante a secagem nas condições experimentais estudadas.

A partir dos resultados obtidos podese inferir que o modelo matemático de Midilli apresentou melhor ajuste aos dados experimentais e foi selecionado para representar o processo de secagem das folhas de jenipapo. Além disso, aumentando a temperatura do ar de secagem houve redução no tempo necessário para que as folhas alcançassem o equilíbrio higroscópico e o 
coeficiente de difusão efetivo aumentou em resposta à elevação da temperatura do ar de secagem. A energia de ativação para a difusão líquida, durante a secagem das folhas de jenipapo, nas condições testadas, foi de $33,87 \mathrm{~kJ} \mathrm{~mol}^{-1}$.

\section{REFERÊNCIAS}

AFONSO JÚNIOR, P.C.; CORRÊA, P.C. Comparação de modelos matemáticos para descrição da cinética de secagem em camada fina de sementes de feijão. Revista Brasileira de Engenharia Agrícola e Ambiental, v.3, n.3, p.349-353, 1999.

AKPINAR, E.K. Mathematical modelling of thin layer drying process under open sun of some aromatic plants. Journal of Food Engineering, v.77, n.4, p.864-870, 2006.

ANDRADE, E.T. et al. Cinética de secagem do café cereja, bóia e cereja desmucilado, em quatro diferentes tipos de terreiros. Revista Brasileira de Armazenamento Especial Café, v.1, n.7, p.37-43, 2003.

ARSLAN, D.; MUSA ÖZCAN, M. Evaluation of drying methods with respect to drying kinetics, mineral content and colour characteristics of rosemary leaves. Energy Conversion and Management, v.49, n.5, p.1258-1264, 2008.

ASAE - American Society of Agricultural Engineers. Standards Engineering Practices Data: Moisture measurement-forages, ASAE S358.2 DEC99. St. Joseph: American Society of Agricultural Engineers, 2000. p.565-572.

BERBERT, P. A. et al. Simulation of coffee drying in a fixed bed with periodic airflow reversal. Journal of Agricultural Engineering Research, v.60, n.3, p.167173, 1995.

BROOKER, D.B.; BAKER-ARKEMA, F.W.; HALL, C.W. Drying and storage of grains and oil seeds. Springer Science \& Business Media, New York: AVI Book Publisher, 1992. 450p.

DIAS, R.A.L. et al. Secagem e Extração de Taninos Totais da Hortelã (Mentha x vilosa Hudson) Drying. Revista Agrarian, v. 4, n. 12, p. 123-133, 2011.

DOYMAZ, İ. Drying of Thyme (Thymus Vulgaris L.) and Selection of a Suitable Thin-Layer Drying Model. Journal of Food Processing and Preservation, v.35, n.4, p.458-465, 2011.

DOYMAZ, İ. Thin-layer drying behaviour of mint leaves. Journal of Food Engineering, v.74, n.3, p.370-375, 2006.

DOYMAZ, İ. Thin-Layer Drying of Bay Laurel Leaves (Laurus nobilis L.). Journal of Food Processing and Preservation, v.38, n.1, p.449-456, 2014.

ERBANO, M.; DUARTE, M.R. Morfoanatomia de folha e caule de Genipa americana L., Rubiaceae. Revista Brasileira de Farmacognosia, v.20, n.6, p. 825-832, 2010.

GONELI, A.L.D. et al. Modelagem matemática e difusividade efetiva de folhas de aroeira durante a secagem. Pesquisa agropecuária tropical, v.44, n.1, p.56-64, 2014.

HENDERSON, S.M. Progress in developing the thin layer drying equation. Transactions of the ASAE, v.17, n.6, p.1167-1168, 1974.

HENDERSON, S.M.; PABIS, S. Grain drying theory. Temperature effect on drying coefficient. Journal of Agricultural Engineering Research, n.6, n.??, p.169174, 1961.

KARATHANOS, V.T. Determination of water content of dried fruits by drying kinetics. Journal of Food Engineering, v.39, n.4, p.337-44, 1999.

KASHANINEJAD, M. et al. Thin-layer drying characteristics and modeling of pistachio nuts. Journal of Food Engineering, v.78, n.1, p.98-108, 2007.

LEWIS, W.K. The drying of solid materials. Journal Industrial Engineering, v.13, n.5, p.427-33, 1921.

LORENZI, H. Árvores brasileiras: Manual de identificação e cultivo de plantas arbóreas nativas do Brasil. Volume 1. $5^{a}$ Edição. Nova Odessa: Plantarum, 2008. 384p.

LORENZI, H.; MATOS, F.J.A. Plantas medicinais no Brasil: Nativas e exóticas. $2^{a}$ Edição, Nova Odessa: Instituto Plantarum, 2008. 544p.

MADAMBA, P.S. et al. Enthalpy-entropy compensation models for sorption and browning of garlic. Journal of Food Engineering, v.28, n.1, p.109-119, 1996.

MARTINAZZO, A.P. et al. Análise e descrição matemática da cinética de secagem de folhas de capim-limão. Revista Brasileira de Engenharia Agrícola e Ambiental, v.11, n.3, p.301-306, 2007.

MARTINAZZO, A.P. et al. Modelagem matemática e parâmetros qualitativos da secagem de folhas de capim- limão [Cymbopogon citratus (DC.) Stapf]. Revista Brasileira de Plantas medicinais, v.12, n.4, p.488-498, 2010.

MIDILLI, A. et al. A new model for single-layer drying. Drying technology, v.20, n.7, p.1503-1513, 2002.

MOHAPATRA, D.; RAO, P.S. A thin layer drying model of parboiled wheat. Journal of Food Engineering, v.66, n.4, p.513-18, 2005.

MOHSENIN, N.N. Physical properties of plant and animal materials. 2. ed. Amsterdam: Gordon and Breach Publishers, 1986, 841 p.

OLIVEIRA, D.E.C. et al. Drying kinetics of Aristolochia cymbifera Mart . and Zucc . leaves. African Journal of Agricultural Research, v.8, n.10, p.922-929, 2013.

PAGE, G.E. Factors influencing the maximum rates of air drying shelled corn in thin layers. Indiana: 1949. Dissertação (Mestrado).Purdue University, USA.

PARK, K.J. et al. Evaluation of drying parameters and desorption isotherms of garden mint leaves (Mentha crispa L.). Journal of Food Engineering, v.51, n.3, p.193-199, 2002.

PRATES, M.F.O. et al. Cinética de secagem de folhas de Solanum lycocarpum A. St.-Hil. (fruta-de-lobo). Revista Brasileira de Plantas medicinais, v.14, n.3, p.514-521, 2012.

RADÜNZ, L.L. et al. Avaliação da cinética de secagem de carqueja. Engenharia na agricultura, v.19, n.1, p.19-27, 2011.

REIS, R.C. et al. Cinética de secagem de folhas de manjericão (Ocimum basilicum L .) via infravermelho. Revista Brasileira de Engenharia Agrícola e Ambiental, v.16, n.12, p.1346-1352, 2012.

RESENDE, O. et al. Modelagem matemática do

Rev. Bras. PI. Med., Campinas, v.17, n.4, supl. II, p.953-963, 2015. 
processo de secagem de duas variedades de feijão (Phaseolus vulgaris L.). Revista Brasileira de Produtos Agroindustriais, v.10, n.1, p.17-26, 2008. RODRÍGUEZ, J. et al. Modelling drying kinetics of thyme (Thymus vulgaris L.): theoretical and empirical models, and neural networks. Food science and technology international, v.20, n.1, p.13-22, 2014.

SHARAF-ELDEEN, Y.I.; BLAISDELL, J.L.; HAMDY, M.Y. A model for ear corn drying. Transactions of the ASAE, v.23, n.5, p.1261-1265, 1980.

SOUZA, R.K.D et al. Aspectos etnobotânicos, fitoquímicos e farmacológicos de espécies de Rubiaceae no Brasil. Revista Cubana de Plantas Medicinales, v.18, n.1, p.140-156, 2013.

THOMPSON, T.L. et al. Mathematical simulation of corn drying: A new model. Transactions of ASAE, v.11, n.4, p.582-586, 1968.

VERMA, L.R. et al. Effects of drying air parameters on rice drying models. Transactions of the ASAE, v.28, n.1, p.296-301, 1985.

WANG, C Y.; SINGH, R. P. Use of variable equilibrium moisture content in modeling rice drying. Transaction of ASAE, v.11, n.78, p.668-672, 1978.

YAGCIOGLU, A.; DEGIRMENCIOGLU, A.; CAGATAY, F. Drying characteristics of laurel leaves under different conditions. In: BAS CETINCELIK A, (ed.) Proceedings of the seventh international congress on agricultural mechanization and energy, 26-27 May, Adana, Turkey. Faculty of Agriculture, Cukurova University; 1999. p. 565-569. 